\title{
Typhoid fever: clinical presentation and associated factors in febrile patients visiting Shashemene Referral Hospital, southern Ethiopia
}

\author{
Limenih Habte ${ }^{1}$, Endale Tadesse ${ }^{2}$, Getachew Ferede ${ }^{3^{*}}$ and Anteneh Amsalu ${ }^{3}$
}

\begin{abstract}
Objective: Although typhoid fever is a major public health problem in Ethiopia, data is not available in the study area. Therefore, this study aimed to determine the prevalence, clinical presentation at the time of diagnosis and associated factors of typhoid fever among febrile patients visiting Shashemene Referral Hospital, southern Ethiopia. A cross-sectional study was conducted from January 1, 2016, to October 30, 2016. Socio-demographic and clinical data were collected using a structured questionnaire. A blood sample was collected and inoculated into Tryptic soy broth.

Results: A total of 421 adult febrile patients suspected of typhoid fever were included in the study. Of these, the overall prevalence of culture-confirmed typhoid fever was 5.0\% (21/421). The prevalence of typhoid fever was significantly associated with rural residence (8.4\%). As compared to the urban resident, the rural resident was 3.6 times more likely found to have culture-confirmed typhoid fever. The prevalence of typhoid fever was significantly associated with those patients whose water source was spring 7 (12.3\%) and river 7 (13.2\%). All of those study participants who used treated water were culture negative. Fever for $\geq 5$ days, abdominal pain, and skin rash independently predicted blood culture-confirmed typhoid fever.
\end{abstract}

Keywords: Blood culture, Typhoid fever, Prevalence, Ethiopia

\section{Introduction}

Typhoid fever is a major public health problem in lowincome and middle-income countries (LMICs) like Ethiopia where there are substandard hygiene and unsafe drinking water supplies and the quality of life is poor [1-5]. Typhoid fever is a systemic infection caused by human-specific food and water-borne pathogens, such as Salmonella enterica subspecies, enterica serovar typhi (S. typhi) or by the related but less virulent Salmonella paratyphi A, B, and C, collectively called typhoidal Salmonella [6]. It is transmitted by the fecal-oral route through contaminated water [4] and food [2, 7]. In 2010, the estimated global episodes of typhoid fever ranged

\footnotetext{
*Correspondence: get29f@gmail.com

${ }^{3}$ Department of Medical Microbiology, University of Gondar, P.O.Box 196 Gondar, Ethiopia

Full list of author information is available at the end of the article
}

from 13.9 to 26.9 million cases [8]. In the same year, the estimated number of typhoid fever cases in LMICs after adjusting for water-related risk was 11.9 million cases with 129, 000 deaths that suggested a higher incidence in Africa [1]. Although national surveillance studies are lacking in Ethiopia, the individual study reported $4.1 \%$ [9] prevalence of typhoid fever among patients with febrile illnesses. Moreover, a systematic and meta-analysis study in Ethiopia showed typhoidal Salmonella (S. typhi) accounted for $42.1 \%$ of the total isolates of Salmonella species reported from 1974 to 2006 years indicating typhoid fever is endemic in Ethiopia [10].

The clinical presentation of typhoid fever varies from a mild illness with low grade fever, headache, fatigue, malaise, loss of appetite, cough, constipation and skin rash or rose spots to in some cases, a fatal complications such as intestinal perforations, gastrointestinal 
hemorrhages, encephalitis and cranial neuritis [11, 12]. Despite its clinical importance, laboratory diagnosis of typhoid fever in resource-limited countries widely depends on non-specific clinical presentation and Widal test which has a low specificity and positive predictive value (PPV) [9]. Hence, leads to wrong diagnoses and over antibiotic prescription [13] that further increases the emergence of multidrug-resistant strains for commonly used drugs [14]. However, diagnosis of typhoid fever using blood culture is the mainstay of diagnosis and has good specificity and can proceed to antibiotic sensitivity test [15]. Yet, blood culture has its own limitation; such as long turnaround time, expensive and needs microbiology experts, due to this it is not routinely performed in resource-limited countries.

Considering typhoid fever is endemic in Ethiopia and the prevalence of this disease varies with demographic, environmental and climatic data; updated information regarding the epidemiology of typhoid fever in the study area may aid policy makers to design appropriate intervention strategies. So far there is no data describing the epidemiology of typhoid fever in the study area. Therefore, the main objective of this study was to determine the prevalence of typhoid fever, clinical presentation at the time of diagnosis and associated factors among febrile patients visiting Shashemene Referral Hospital, southern Ethiopia.

\section{Main text}

Materials and methods

\section{Study design, study area and study period}

A hospital-based cross-sectional study was conducted in Shashemene Referral Hospital from January 1, 2016, to October 30, 2016. Shashemene is the town of West Arsi zone in Oromia regional state, Ethiopia.

\section{Study population and sample size}

All adult patients (age $\geq 18$ years) suspected of typhoid fever who visited the hospital during the study period were invited to participate in the study. The sample size was estimated to be 384 by using single proportion formula at $95 \%$ confidence interval, assuming typhoid prevalence of $50 \%$ and $5 \%$ marginal error; by adding $10 \%$ contingency a total sample size was 422 . Participants already on antibiotic treatment within 2 weeks and diagnosed with other known febrile illness were excluded from the study.

Typhoid fever suspected patients are defined as patients (axillary temperature, $\geq 38{ }^{\circ} \mathrm{C}$ ) who reported having a fever for at least 3 days and headache. In addition, if there was clinical suspicion of typhoid fever by the attending senior clinical staff at the study sites.
Typhoid fever confirmed cases were defined as a patient with fever (axillary temperature, $\geq 38^{\circ} \mathrm{C}$ ) for at least 3 days with a laboratory-confirmed positive blood culture of $S$. typhi.

\section{Data collection}

Socio-demographic and clinical data All adult febrile patients visiting adult outpatient department (OPD) clinic were clinically examined by the physicians and those suspected of typhoid fever were requested for blood culture. After obtaining written informed consent socio-demographic and clinical data were collected by nurses using a structured questionnaire that was validated and edited after small pilot study.

Blood sample collection and processing In the laboratory, a total of $5 \mathrm{ml}$ blood sample was collected aseptically using $70 \%$ alcohol and $2 \%$ tincture of iodine from a peripheral vein in each patient. Then the blood sample was dispensed into a sterile bottle containing $45 \mathrm{ml}$ of Tryptic soy broth culture medium (Becton, DickinsonUSA).

Isolation and identification of bacteria The inoculated bottles were incubated aerobically at $37^{\circ} \mathrm{C}$ for 7 days in the Microbiology laboratory and observed for a sign of bacterial growth (turbidity, hemolysis, air bubbles or gas production and clot formation) on the daily bias for up to 7 days. Bottles which showed sign of growth were further processed by Gram stained and sub-cultured on MacConkey agar (Park Scientific Unlimited-England), at $37{ }^{\circ} \mathrm{C}$ for 24-h. The plate was then aerobically incubated for $18-24$ h at $37^{\circ} \mathrm{C}$. A blood sample containing broth with no bacterial growth after 7 days were sub-cultured on blood agar before being reported as a negative result. Identification of isolates were done by colony morphology, Gram staining, and biochemical tests using Kligler iron agar (KIA)-(Becton, Dickinson-USA), Motility, Indole, Ornitine (MIO) (Park Scientific Unlimited-England), Citrate Utilization test (OXID LTD England), Urease test (Mast Group Ltd, UK) and lysine Iron agar (LIA)-(LiofilchemItaly) test.

\section{Data analysis}

Data were entered and analyzed using SPSS version 20 software. Chi square and a logistic regression model were used to determine the predictors of typhoid fever. Adjusted odds ratio (AOR) with 95\% CI was also computed from multivariable logistic regression adjusting for possible confounders. A p-value of less than 0.05 was considered statistically significant. 


\section{Results}

Socio-demographic characteristics of study participants

All 422 study participants who were suspected of typhoid fever by physicians during a study period were approached to the study. However, one study participant's data was found incomplete due to this, a total of 421 adult febrile patients suspected of typhoid fever were included. The mean (standard deviation [SD]) of the participants' age was 35.3 (11.3) years, ranged from 18 to 66 years. More than half of the patients 235 (55.8\%) were females, $138(32.8 \%)$ were in the age category of $31-40$ years and $243(57.7 \%)$ were an urban resident. The majority 254 (60.3\%) were married, 177 (42\%) farmer, 174 (41.3\%) had no formal education and 333 (79.1\%) had on the average monthly income of less than or equal to 1000 Ethiopian Birr (ETB) (Table 1).
The overall prevalence of culture-confirmed typhoid fever was $5.0 \%$ (21/421, 95\% CI 2.9-7.1\%). Rural residence showed a statistically significant association with the prevalence of typhoid fever ( $p$-value $<0.05$ ) while age, sex, marital status, occupation, educational status and monthly income were not significantly associated. Those study participants who were lived in rural areas were 3.6 times more likely found to have culture-confirmed typhoid fever $(\mathrm{COR}=3.6,95 \%$ CI 1.38-9.57) as compared to the urban resident. Students were 5.7 times $(\mathrm{COR}=5.7,95 \%$ CI $0.64-50.0)$ more likely to have culture-confirmed typhoid fever as compared with an employee (Table 1).

Table 1 The prevalence of typhoid fever with respect to socio-demographic characteristics among febrile patients visiting Shashemene Referral Hospital, southern Ethiopia, 2016

\begin{tabular}{|c|c|c|c|c|}
\hline Variables & Total $(\mathrm{N}=421)$ & Culture positive $(\mathrm{N}=21)$ & COR $(95 \% \mathrm{Cl})$ & p-value \\
\hline \multicolumn{5}{|l|}{ Age (years) } \\
\hline $18-20$ & $40(9.5)$ & $3(7.5)$ & $2.7(0.58-12.67)$ & 0.204 \\
\hline $21-30$ & $129(30.6)$ & $8(6.2)$ & $2.2(0.65-7.54)$ & 0.203 \\
\hline $21-30$ & $138(32.8)$ & $4(2.9)$ & 1 & \\
\hline $41-50$ & $74(17.6)$ & $3(4.1)$ & $1.4(0.31-6.50)$ & 0.655 \\
\hline$>50$ & $40(9.5)$ & $3(7.5)$ & $2.7(0.58-12.67)$ & 0.204 \\
\hline \multicolumn{5}{|l|}{ Sex } \\
\hline Male & $186(44.2)$ & $9(4.8)$ & 1 & \\
\hline Female & $235(55.8)$ & $12(5.1)$ & $1.1(0.43-2.56)$ & 0.90 \\
\hline \multicolumn{5}{|l|}{ Residence } \\
\hline Urban & $243(57.7)$ & $6(2.5)$ & 1 & \\
\hline Rural & $178(42.3)$ & $15(8.4)$ & $3.6(1.38-9.57)$ & 0.009 \\
\hline \multicolumn{5}{|l|}{ Marital status } \\
\hline Married & $254(60.3)$ & $10(3.9)$ & 1 & \\
\hline $\mathrm{D} / \mathrm{W}$ & $97(23.1)$ & $7(7.2)$ & $1.9(0.70-5.13)$ & 0.207 \\
\hline Unmarried & $70(16.6)$ & $4(5.7)$ & $1.5(0.45-4.87)$ & 0.520 \\
\hline \multicolumn{5}{|l|}{ Occupation } \\
\hline Farmer & $177(42.0)$ & $10(5.6)$ & $3.6(0.45-28.7)$ & 0.227 \\
\hline Merchant & $125(29.7)$ & $5(4.0)$ & $2.5(0.27-21.9)$ & 0.408 \\
\hline Students & $58(13.8)$ & $5(8.6)$ & $5.7(0.64-50.0)$ & 0.119 \\
\hline Employee & $61(14.5)$ & $1(1.6)$ & 1 & \\
\hline \multicolumn{5}{|l|}{ Educational status } \\
\hline No-formal & $174(41.3)$ & $12(6.9)$ & $2.1(0.72-6.12)$ & 0.172 \\
\hline Primary & $100(23.8)$ & $4(4.0)$ & $1.2(0.31-4.52)$ & 0.806 \\
\hline Secondary and above & $147(34.9))$ & $5(3.4)$ & 1 & \\
\hline \multicolumn{5}{|l|}{ Monthly } \\
\hline$\leq 1000$ & $333(79.1)$ & $18(5.4)$ & $1.6(0.47-5.6)$ & 0.448 \\
\hline \multicolumn{5}{|l|}{ Income (Birr) } \\
\hline$>1000$ & 88 (20.9) & $3(3.4)$ & 1 & \\
\hline
\end{tabular}

$D / W$ divorce/widowed 


\section{Clinical presentation at the time of diagnosis}

Concerning their clinical presentation at the time of diagnosis, all the participants were febrile for at least 3 days and had a headache. The majority $358(85.0 \%)$ presented with fever less than 5 days, fatigue $348(82.7 \%)$ and loss of appetite $273(64.8 \%)$; whereas $76(18.1 \%), 120(28.5 \%)$, 65 (15.4\%), 187 (44.4\%) and 8 (1.9\%) presented with constipation, diarrhea, abdominal pain, cough and skin rash, respectively. Fever, abdominal pain, and skin rash showed a statistically significant association with the prevalence of typhoid fever while fatigue, loss of appetite, constipation, diarrhea, and cough were not significantly associated. Febrile patients who presented with a fever greater or equal to 5 days was 18.2 times (AOR $=18.2,95 \% \mathrm{CI}$ 6.45-54.5) more likely to have culture-confirmed typhoid fever as compared to patients presented with fever of less than 5 days. Febrile patients presented with abdominal pain was 3.9 times $(\mathrm{AOR}=3.9,95 \% \mathrm{CI} 1.32-11.23)$ and skin rash was 11.1 times (AOR $=11.1,95 \%$ CI 1.61-76.2) higher odds of culture-confirmed typhoid fever as compared to those who hadn't have abdominal pain and skin rash respectively (Table 2 ).

\section{Source of food and water}

The majority of the study participants 358 (85\%) usually consume homemade food and 253 (60.1\%) obtained water supplies from the pipe. The analysis of the prevalence of typhoid fever showed a statistically significant association with the source of water (p-value $<0.05$ ), while the source of food was not showed a statistically significant association. None of the study participants who used treated water was culture positive (Table 3 ).

\section{Discussion}

In this study, the prevalence of typhoid fever was 5.0\% concordant with studies conducted in Central Ethiopia (4.1\%) [9] among febrile patients with symptoms clinically similar to typhoid fever and outside of Ethiopia such as: in Kenya among adult patients (6.3\%) [16] and Papua New Guinea among all age group (4\%) [17]. However, our finding was higher than a study conducted in Mekelle, Ethiopia (1.6\%) [13] and lower than a study conducted in Egypt (13.64\%) [18]. The differences could be explained by the difference in a geographical location where participants in our study

Table 2 Clinical signs and symptoms of typhoid fever and their association with blood culture positivity among adult patients visiting Shashemene Referral Hospital, southern Ethiopia, 2016

\begin{tabular}{|c|c|c|c|c|c|}
\hline Variable $(n=421)$ & Total N (\%) & Culture pos N (\%) & COR $(95 \% \mathrm{Cl})$ & AOR $(95 \% \mathrm{Cl})$ & P-value \\
\hline \multicolumn{6}{|l|}{ Fatigue } \\
\hline Yes & $348(82.7)$ & $17(4.9)$ & $1.1(0.37-3.46)$ & & \\
\hline No & $73(17.3)$ & $4(5.5)$ & 1 & & \\
\hline \multicolumn{6}{|l|}{ Fever } \\
\hline$<5$ days & $358(85.0)$ & $6(1.7)$ & 1 & 1 & \\
\hline$\geq 5$ days & $63(15.0)$ & $15(23.8)$ & $18.3(6.78-49.5)^{*}$ & $18.2(6.45-54.5)$ & 0.001 \\
\hline \multicolumn{6}{|l|}{ Loss of appetite } \\
\hline Yes & $273(64.8)$ & $13(4.8)$ & 1 & & \\
\hline No & $148(35.2)$ & $8(5.4)$ & $1.1(0.46-2.82)$ & & \\
\hline \multicolumn{6}{|l|}{ Constipation } \\
\hline Yes & $76(18.1)$ & $7(9.2)$ & $2.4(0.93-6.16)$ & & \\
\hline No & $345(81.9)$ & $14(4.1)$ & 1 & & \\
\hline \multicolumn{6}{|l|}{ Diarrhea } \\
\hline Yes & $120(28.5)$ & $6(5.0)$ & $1.0(0.38-2.65)$ & & \\
\hline No & $301(71.5)$ & $15(5.0)$ & 1 & & \\
\hline \multicolumn{6}{|l|}{ Abdominal pain } \\
\hline Yes & $65(15.4)$ & $8(12.3)$ & $3.7(1.47-9.33)^{*}$ & $3.9(1.32-11.23)$ & 0.013 \\
\hline No & $356(84.6)$ & $13(3.7)$ & 1 & & \\
\hline \multicolumn{6}{|l|}{ Cough } \\
\hline Yes & $187(44.4)$ & $11(5.9)$ & $1.4(0.58-3.37)$ & & \\
\hline No & $234(55.6)$ & $10(4.3)$ & 1 & & \\
\hline \multicolumn{6}{|l|}{ Skin rash } \\
\hline Yes & $8(1.9)$ & $3(37.5)$ & $13.2(2.92-59.4)^{*}$ & $11.1(1.61-76.2)$ & 0.015 \\
\hline No & $413(98.1)$ & $18(4.4)$ & 1 & 1 & \\
\hline
\end{tabular}


Table 3 Sources of drinking water and food in typhoid fever suspected patients visiting Shashemene Referral Hospital from January to October 2016

\begin{tabular}{lrll}
\hline Variable & Total N (\%) & $\begin{array}{l}\text { Culture } \\
\text { positive N (\%) }\end{array}$ & $\begin{array}{l}\text { Chi square } \\
\text { (p-value) }\end{array}$ \\
\hline Source of water & & & $\begin{array}{c}X^{2}=19.6, d f=3 \\
(0.0001)\end{array}$ \\
Pipe & $253(60.1)$ & $7(2.8)$ & \\
Spring & $56(13.3)$ & $7(12.5)$ & \\
River & $53(12.6)$ & $7(13.2)$ & \\
Treated (Wuha agar) & $59(14.0)$ & 0 & $X^{2}=0.51, d f=1$ \\
Source of food & & & $(0.473)$ \\
& & & \\
Homemade & $358(85.0)$ & $19(5.3)$ & \\
Hotel & $63(15.0)$ & $2(3.2)$ & \\
\hline
\end{tabular}

particularly rural residents are living close to rivers, and they used river water for drinking that might tend to have more typhoid risk $[19,20]$.

Although studies reported that there is no significant difference on the occurrence of typhoid between urban and rural environments [20], in this study rural residents were associated with higher risk of typhoid fever as compared to urban residents. This could be explained by suboptimal access to safe water and lack of hygienic education which was supported by the high prevalence of typhoid among farmers with no formal education. In addition, lack of toilet and/or hand washing practice after toilet, open defecation practices near to the springs and rivers and inadequate medical care are common in the rural residence that may serve as a carrier for transmission [20]. In this study, the high odds of typhoid fever among students as compared to the employee is perhaps as a result of lack of sufficient safe drinking water, toilet and water for hand washing after the toilet in the educational institutions. This probably creates a greater 'opportunity' for person-to-person transmission in these congregated sites.

The current study assessed the patterns of clinical presentation to help in case identification at presentation to health facilities where there is no confirmatory microbiological test and it was found that patients having a fever for greater than or equal to 5 days, abdominal pain and skin rash had a significant association with culture-confirmed typhoid fever. This is in accordance with the World Health Organization (WHO) guideline in that the most common manifestations of untreated typhoid fever in the first week of infection was stepped ladder fever pattern or insidious onset fever, skin rash, anorexia, mild cough and constipation [12].

\section{Conclusions}

The study illustrated that typhoid fever was one of the causes of a significant amount of morbidity in rural communities. To confirm typhoid fever suspected cases using blood culture, considering patients clinical presentation such as duration of fever $\geq 5$ days, abdominal pain and skin rash could decrease the time and cost associated with the diagnosis. Moreover, ensuring access to safe water and delivering health education to drink treated water particularly for rural residents could reduce typhoid fever transmission.

\section{Limitations}

This study has some limitations in light of which results need to be interpreted. First, as a hospital-based study that enrolled participants in the outpatient department, typhoid patients that don't seek healthcare at the Shashamane Referral Hospital were missed. Second, identification of species by anti-sera and antibiotic susceptibility test for isolated pathogen was not done. Third, access to safe water, toilet, environmental sanitation and occurrence of the outbreak was not assessed during the study period. But, the study was conducted almost throughout the year, relatively in larger sample size and use of the gold standard method for diagnosis may ensure the quality of the generated data.

\section{Abbreviations \\ AOR: adjusted odds ratio; ATCC: American Type Culture Collection; COR: crude odds ratio; LMICs: low-income and middle-income countries; OPD: outpatient department; OR: odds ratio; PPV: positive predictive value; SD: standard devia- tion; WHO: World Health Organization; S. typhi: Salmonella typhi.}

\section{Authors' contributions}

LH was the primary researcher, conceived the study, designed and collected data. ET contributed designing the study, analysis, and interpretation of data and reviewed the initial draft manuscript. GF contributed data analysis, interpretation, and write-up the final manuscript for publication. AA contributed designing the study, data analysis and interpretation, drafted the manuscript for publication. All authors read and approved the final manuscript.

\section{Author details \\ ${ }^{1}$ Shashemene Referral Hospital, Oromia Region, Shashemene, Ethiopia. \\ ${ }^{2}$ Department of Medical Laboratory Sciences, Hawassa University, Hawassa, Ethiopia. ${ }^{3}$ Department of Medical Microbiology, University of Gondar, P.O.Box 196, Gondar, Ethiopia.}

\section{Acknowledgements}

First, we would like to thank the study participants for their voluntary participation. Second, we thank the staffs of Shashemene Referral Hospital for their technical support during data collection and providing consumable materials. We would also extend our gratitude to Adama Regional Laboratory for their provision of culture media and positive control strains. Last but not the least, we express our sincere appreciation to Mr. Daba Ejara for his support in every aspect during the process of the study.

\section{Competing interests}

The authors declare that they have no competing interests. 


\section{Availability of data and materials}

The datasets analyzed in this study are available from the corresponding author on reasonable request.

\section{Consent to publish}

Not applicable.

\section{Ethics approval and consent to participate}

Ethical approval was obtained from the Institutional Review Board (IRB) of Hawassa University, College of Medicine and Health Sciences. Written informed consent was obtained from each study participants. The laboratory results were communicated to their attending physicians for appropriate treatment.

\section{Funding}

Funding is not applicable.

\section{Publisher's Note}

Springer Nature remains neutral with regard to jurisdictional claims in published maps and institutional affiliations.

Received: 5 July 2018 Accepted: 16 August 2018

Published online: 22 August 2018

\section{References}

1. Mogasale V, Maskery B, Ochiai RL, Seok Lee J, Mogasale W, Ramani E, Eun Kim Y, Kyung Park J. WierzbaTF: burden of typhoid fever in low-income and middle-income countries: a systematic, literature-based update with risk-factor adjustment. Lancet Glob Health. 2014;2:e570-80.

2. Mama M, Alemu G. Prevalence, antimicrobial susceptibility patterns and associated risk factors of Shigella and Salmonella among food handlers in Arba Minch University, South Ethiopia. BMC Infect Dis. 2016;16:686.

3. Zeru K, Kumie A. Sanitary conditions of food establishments in Mekelle town, Tigray, north Ethiopia. Ethiop J Health Dev. 2007;21(1):3-11.

4. Farooqui A, Khan A, Kazmi SU. Investigation of a community outbreak of typhoid fever associated with drinking water. BMC Public Health. 2009;9:476.

5. Kanungo S, Dutta S, Sur D. Epidemiology of typhoid and paratyphoid fever in India. J Infect Dev Ctries. 2008;2(6):454-60.

6. Sattar AA, Jhora ST, Yusuf MA, Islam MB, Islam MS, Roy S. Epidemiology and clinical features of typhoid fever: burden in Bangladesh. J Sci Found. 2012;10(1):38-49.

7. Hosoglu S, Selen MK, Geyik MF, Akalin S, Ayaz C, Acemoglu H, Loeb M. Risk factors for typhoid fever among adult patients in Diyabakir, Turkey. Epidemiol Infect. 2006;134(3):612-6.
8. Buckle GC, Walker CL, Black RE. Typhoid fever and paratyphoid fever: systematic review to estimate global morbidity and mortality for 2010. J Glob Health. 2012:2(1):1-8.

9. Andualem G, Abebe T, Kebede N, Gebre-Selassie S, Mihret A, Alemayehu $\mathrm{H}$. A comparative study of Widal test with blood culture in the diagnosis of typhoid fever in febrile patients. BMC Res Notes. 2014;7:653.

10. Tadesse G. Prevalence of human Salmonellosis in Ethiopia: a systematic review and meta-analysis. BMC Infect Dis. 2014;14:88.

11. Mogasale V, Desai SN, Mogasale V, Park JK, Ochiai RL, Wierzba TF. Case fatality rate and length of hospital stay among patients with typhoid intestinal perforation in developing countries: a systematic literature review. PLoS ONE. 2014;9:e93784.

12. World Health Organization. Guidelines for the management of typhoid fever. WHO; 2011.

13. Gebreyesus AW, Negash LW, Aregawi SG, Luel AW, Muthupandian S, Dejenie TH. AsmelashTD: diagnosis and treatment of typhoid fever and associated prevailing drug resistance in northern Ethiopia. Int J Infect Dis. 2015:35:96-102.

14. Andualem G, Abebe T, Kebede N, Gebre-Selassie S, Mihret A, Alemayehu $H$. Prevalence and antimicrobial susceptibility patter of Salmonella typhi and Salmonella paratyphi isolates from patients with clinical symptom compatible with typhoid fever. In: 2nd International Congress on Bacteriology and Infectious Diseases. J Bacteriol Parasitol. 2014;5(4):183.

15. World Health Organization. Background document: the diagnosis, treatment and prevention of typhoid fever. WHO; 2003.

16. Benjamin Nguri KA. Risk factors influencing typhoid fever occurrence among the adults in Maina Slum, Nyahururu municipality, Kenya. MSc thesis 2011

17. Siba V, Howood PV, Vanuga K, Wapling J, Sehuko R, Siba PM, Greenhill AR. Evaluation of serological diagnostic tests for typhoid fever in Papua New Guinea using a composite reference standard. Clin Vaccine Immunol. 2012;19(11):1833-7.

18. Hamdy MS, Abdel-Rahman S. Evaluation of enterocheck WB ${ }^{\circledR}$ test in diagnosis of typhoid fever among Egyptian adults. Egypt J Med Microbiol. 2014;23(4):47-50.

19. Tran HH, Bjune G, Nguyen BM, Rottingen JA, Grais RF, Guerin PJ. Risk factors associated with typhoid fever in Son La Province, northern Vietnam. Trans R Soc Trop Med Hyg. 2005;99:819-26.

20. Dewan AM, Corner R, Hashizume M, Onge ET. Typhoid fever and its association with environmental factors in the Dhaka Metropolitan Area of Bangladesh: a spatial and time-series approach. PLoS Negl Trop Dis. 2013;7(1):e1998. https://doi.org/10.1371/journal.pntd.0001998.
Ready to submit your research? Choose BMC and benefit from:

- fast, convenient online submission

- thorough peer review by experienced researchers in your field

- rapid publication on acceptance

- support for research data, including large and complex data types

- gold Open Access which fosters wider collaboration and increased citations

- maximum visibility for your research: over 100M website views per year

At BMC, research is always in progress.

Learn more biomedcentral.com/submissions 